\title{
An Investigation on the Characteristics of Mobile Applications: A Survey Study
}

\author{
Harleen K. Flora \\ The IIS University, Jaipur, India \\ E-mail: harleenflora@gmail.com \\ Dr. Xiaofeng Wang \\ Free University of Bozen/Bolzano, Italy \\ E-mail: xiaofeng.wang@unibz.it \\ Dr. Swati V. Chande \\ International School of Informatics and Management, Jaipur, India \\ E-mail: swati.chande@iisuniv.ac.in
}

\begin{abstract}
Swift advances in mobile communication technology have spawned almost unlimited new mobile applications. Mobile application development is an extremely well growing industry across the globe that created new opportunities of modern businesses and pioneered new technologies in the area. In order to build high quality mobile applications, it is imperative to understand the key characteristics that define mobile applications, which if wisely considered and implemented, can facilitate the delivery of truly exceptional, valuable and user friendly mobile apps that satisfy users' needs. Only few scientific publications can be found which specifically identify the key characteristics and what makes mobile applications different from traditional software. For this purpose, we conducted an online survey from the mobile research and development community. The survey questions covered the entire mobile application development lifecycle starting from inception to the maintenance stage. This paper presents the survey results by classifying the key characteristics that differentiate mobile applications from traditional ones into three categories: Hardware, Software (application interaction, application development, and application security) and Communication. The study contributes towards a greater understanding of mobile software and the current trends in the mobile application development. It also highlights various features and attributes that assist in developing high quality mobile software applications.
\end{abstract}

Index Terms-Mobile Computing, Mobile Characteristics, Mobile Application Development

\section{INTRODUCTION}

In recent years mobile phones are gaining a new momentum with the emergence of smart phones and tablets. Mobile applications (or mobile apps) are compact programs developed to work on smart phones, tablets, and feature phones. A rapid escalation of mobile apps has inspired mobile developers tremendously. There is a continuous increase in the number of mobile applications developed and downloaded every year. And the sudden emergence of mobile devices has made the new computing platform financially beneficial for not only entrepreneurs but also independent software developers.
Mobile application development is quite different from traditional software development. To build a good mobile application, it is crucial to understand the key features that define a good mobile app which, if practically applied, make them useful and valuable. The wide variety of tools and platforms of mobile devices calls for an examination of the unique characteristics of mobile apps and an evaluation of new features and methods for the development process, including designing, coding, testing, deploying and maintaining mobile apps. Without a sufficient understanding of these characteristics, mobile devices may be exposed to prospective attacks which cannot be addressed promptly. However, there is still a lack of research initiatives of different types, categories and characteristics of mobile applications. This observation motivated the study described in the paper.

For this purpose, a web survey was conducted in the current paper involving mobile companies, mobile app development team members, mobile experts, researchers and relevant stakeholders to investigate current emerging practices and new research directions in the area of mobile application development. The paper describes the considerations that developers need to take into account when developing mobile apps, due to hardware, software and communication characteristics specific to those devices. The paper also summarises the key characteristics and features that differentiate the development of mobile applications from conventional desktop applications and concludes with the proposed future work based on the study outlined.

The paper is organized as follows. Section I presents this introduction. Section II focuses on the background and the related work is discussed. Section III explains the research approach used for this study. Section IV describes the findings by classifying the key characteristics that differentiate mobile apps from traditional applications into three categories: characteristics related to Hardware, Software (application interaction, application development, and application 
security) and Communication. Finally, Section V concludes the paper and outlines future work.

\section{BACKGROUND AND RELATED WORK}

\section{A. Mobile Application Types and Categories}

To be successful in mobile application development (MAD), it is imperative to have a profound understanding of what mobile applications really are and how they differ from desktop applications that users also interact with. 'Mobile Application Types' are generally confused with 'Mobile Application Categories'.

Table 1 and Table 2 below describe various 'types' and 'categories' implicated in the development of mobile apps [1]. The two tables are explained using suitable examples so as to clearly differentiate between types and categories.

Table 1. Mobile Application Types

\begin{tabular}{|c|c|c|}
\hline & Type & Description \\
\hline 1 & $\begin{array}{c}\text { Browser Access } \\
\text { Apps }\end{array}$ & $\begin{array}{c}\text { Apps are not installed in the device and can be accessed through native browser by hitting the } \\
\text { URL of the web. The device memory size is not imperative as the app data is not stored in the } \\
\text { device. It is completely dependent on the quality of the browser. For example, m.yahoo.com, } \\
\text { www.google.com. }\end{array}$ \\
\hline 2 & $\begin{array}{c}\text { Native } \\
\text { Apps }\end{array}$ & $\begin{array}{c}\text { Apps are installed in the device. They do not need any data transfer to the server and works in the } \\
\text { device without network as the data about the app is stored in the device itself. For example, } \\
\text { Notes and Reminder in iPhones. }\end{array}$ \\
\hline 3 & Hybrid Apps (Web) & $\begin{array}{c}\text { Apps are installed in the device and always require internet connection to run and function. For } \\
\text { example, Social Networking Apps (Facebook, Twitter), Instant Messengers (Skype), E- } \\
\text { Commerce (Flipkart), Internet Speed Testing (Speedtest). }\end{array}$ \\
\hline 4 & Hybrid Apps (Mixed) & $\begin{array}{c}\text { Apps are installed in the device and may or may not require internet connection to run and } \\
\text { function. For example, Medical apps and few games in that can be played alone, offline and go } \\
\text { online too for playing with multiple players. }\end{array}$ \\
\hline
\end{tabular}

Table 2. Mobile Application Categories

\begin{tabular}{|c|c|c|}
\hline & Categories & Description \\
\hline 1 & Communications & $\begin{array}{l}\text { E-mail clients, IM clients, Social networking clients, mobile/internet browsers, News/Information } \\
\text { clients, on device portals (Java portals) }\end{array}$ \\
\hline 2 & Games & Puzzle/Strategy, Cards/Casino, Action/Adventure, Sports, Leisure Sports \\
\hline 3 & Multimedia & $\begin{array}{c}\text { Graphics/Image viewers, Presentation viewers, Video players, Audio players, Streaming players } \\
\text { (Audio/Video) }\end{array}$ \\
\hline 4 & Productivity & $\begin{array}{c}\text { Calendars, Calculators, Diary, Notepad/Memo/Word Processors, Spreadsheet, Directory Services, } \\
\text { Banking and finance, Call recording, Mobile health monitoring, Mobile advertising }\end{array}$ \\
\hline 5 & Travel & $\begin{array}{c}\text { City guides, Currency convertor, GPS (location based service), Maps, Translators, Itineraries, } \\
\text { Schedules, Weather forecaster }\end{array}$ \\
\hline 6 & Utilities & $\begin{array}{c}\text { Profile Manager, Idle screens, Screensavers, Address book, Task manager, Call manager, File } \\
\text { manager, Mobile search }\end{array}$ \\
\hline 7 & Education & Alphabet, Numerical \\
\hline
\end{tabular}

Various mobile applications that are available on any apps store these days can be categorized into these types and categories.

\section{B. Key Characteristics of Mobile Applications}

There are common concerns to both traditional software engineering and MAD such as hardware device integration, performance, reliability, storage limitations and security issues [2]. However, MAD presents some additional requirements such as potential interaction with other applications, integration with device sensors, native and hybrid (mobile web) applications, families of hardware and software platforms, security requirements, variability of user interfaces, complexity of testing, power consumption, scarce documentation, and short length session activities conducted on mobile devices $[3,4]$.
Mobile based applications have been grown as the development of mobile computing and communication technologies [5]. Divergent hardware makers for mobile phones and tablet platforms have necessitated developers to make a series of the same application tailored for each type of devices. Software reuse in MAD has been recommended as well [6,7].

In addition, the key attributes that define a successful mobile app are functionality, reliability, flexibility, accessibility, portability, efficiency, maintainability, usability and responsiveness, iterated in line with users requirements, the quality characteristics specified in ISO $9126[8,9,10,11,12]$.

The ten customs [13] through which desktop websites differ from mobile sites on the basis of content prioritization (including the most crucial functions and features); vertical instead of horizontal navigation; bars, 
tabs, and hypertext; text and graphics; contextual and global navigation; footers; breadcrumbs; progress indicator; integration with phone functions; and localized $\&$ personalized search.

Mobile computing $[14,15]$ technology enables mobile users to create, access, process, store and communicate information without being constrained to a single location. Powerful mobile computing solutions are possible due to the integrity of extremely powerful and small computing hardware (defined by size and form factor, weight, microprocessor, primary storage, secondary storage, screen size and type, means of input, means of output, battery life, communication capabilities, expandability, durability of the device); specialized system and application software (common system software and operating environments include MSDOS, MS Windows, UNIX, and more) and improved telecommunication (communication with fixed information system categorized as Connected, Weekly Connected, Batch and Disconnected).

Furthermore, the specific mobile app, hardware, software, and operating location/environment determine which communication media are appropriate to use for a mobile computing solution.

The current advancement in mobile applications and different market and technology trends have dramatically benefited developers. They have generated many new development opportunities, to distribute and generate significant revenues with the emerging mobile application portals $[16,17]$.

\section{RESEARCH APPROACH}

A survey was conducted for this study to gain valuable input regarding various emergent practices during the developmental phase of mobile apps. The results of the survey were utilized as a starting point to identify specific requirements in the area of mobile software engineering for new and revolutionary business practices.

An online extensive questionnaire was designed based on the available literature related to mobile app development and the evaluation of several online surveys conducted in the past by various commercial organizations researching on mobile app development. The main contributors to the survey were mobile companies, MAD team members, mobile experts, researchers and relevant stakeholders. They were invited to participate in the survey via mobile developer forums to solicit responses which covered multiple mobile app platforms and development aspects. More than 130 responses were received during the data collection period and the participation in the survey has been entirely voluntary.

The design of the questionnaire was evaluated and validated by several experts in the area before it was handed over to the participants. The data was analysed quantitatively and qualitatively by studying each response individually and then analysing all responses collectively. The validity of the research design and findings was assessed by relevant experts in the area. Since the focus of this paper is on the characteristics of mobile applications, we report the part of the survey results that are pertinent to this focus.

Figures 1 to 5 below represent the general information regarding the demographics and experience of the respondents in the area of mobile application development.

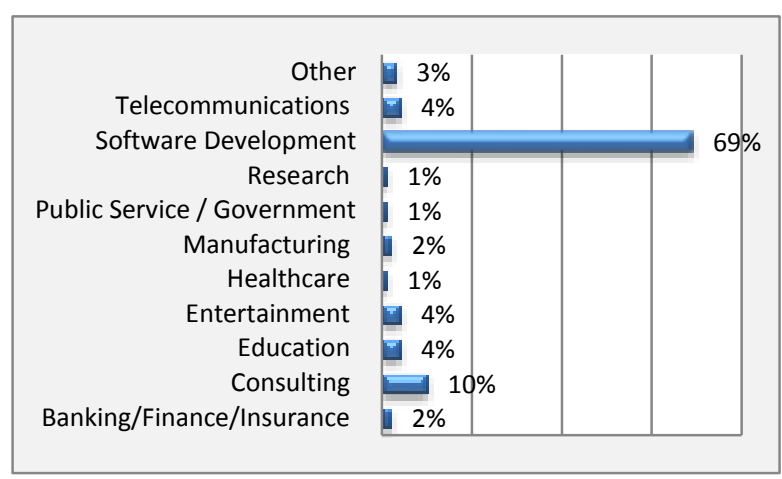

Fig. 1. Organization Type.

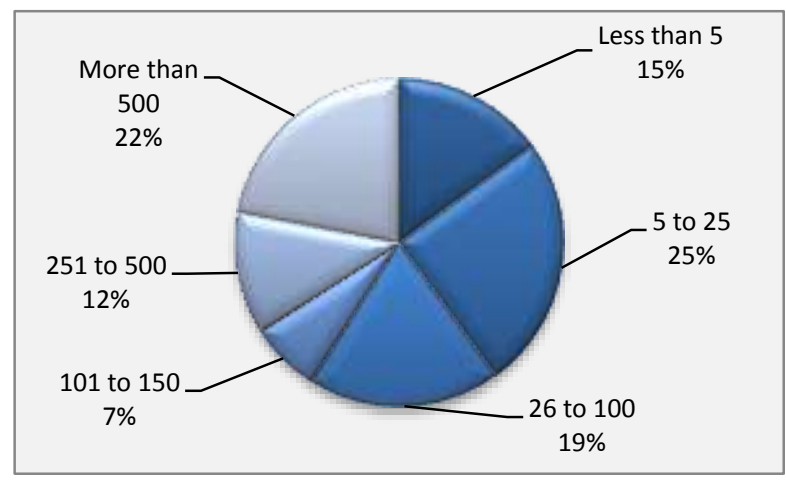

Fig. 2. Organization Size.

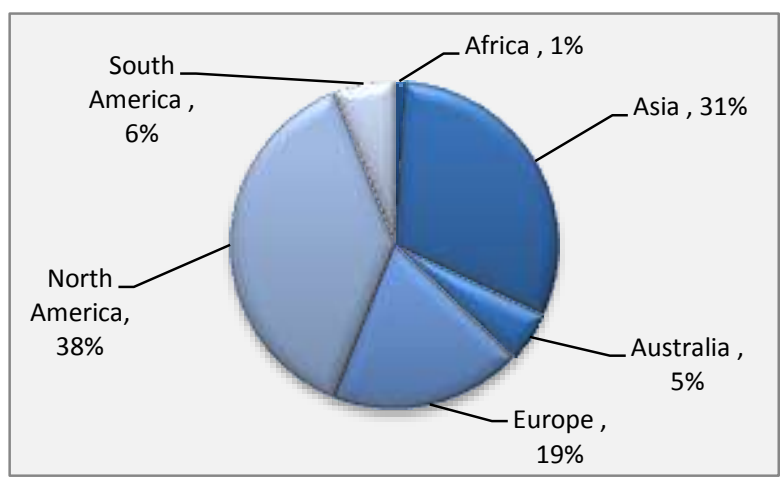

Fig. 3. Organization Location.

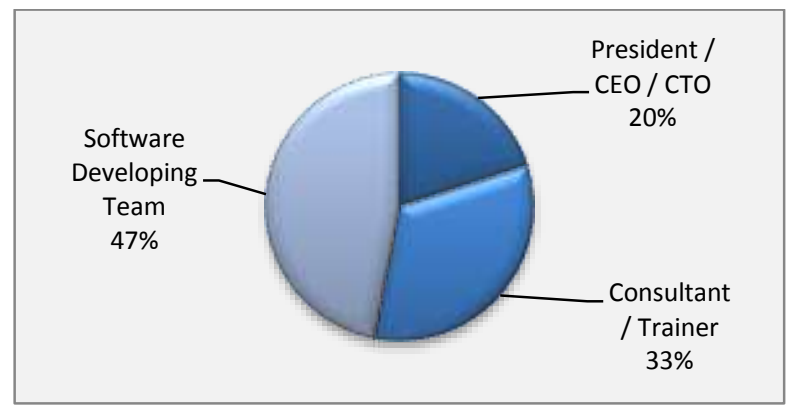

Fig. 4. Respondent Role in Organization. 


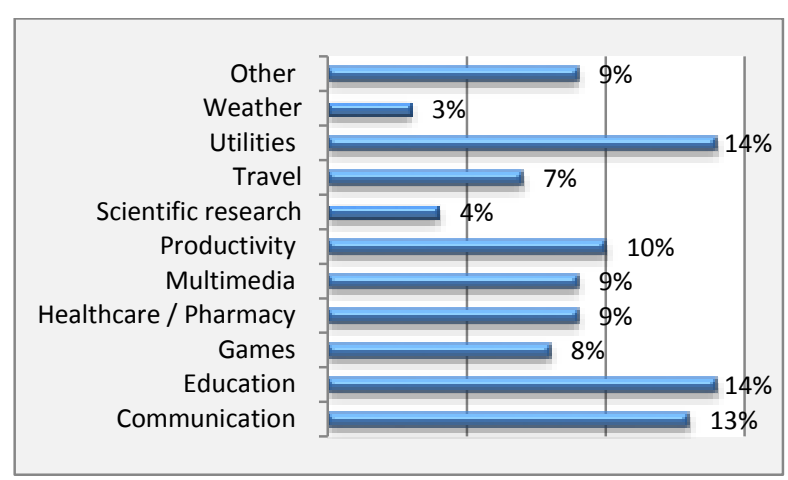

Fig. 5. Categories of Mobile Applications developed.

It can be seen from the figures that $69 \%$ of the respondents come from mobile industry with actual mobile app development experience. Most of the respondents have experience in developing mobile software mainly related to education (14\%), utilities (14\%) and communication (13\%). Maximum responses were received from development team members (47\%) as compared to consultant/trainers $(33 \%)$ and top-level management $(20 \%)$. The respondents cover different continents, with the biggest group (38\%) coming from North America.

\section{FINDINGS}

The majority of the survey respondents are of the opinion that mobile apps significantly vary from their desktop counterparts in several ways despite having notable similarities. Many respondents believe that a mobile phone performs as a personal computer and contains personal information of the owner that is used exclusively by the owner, unlike PCs which may sit in a room and may be shared by various individuals. In addition, the respondents are of the opinion that a mobile phone also acts as an agent, taking messages or notifying the owner upon significant events. Since a mobile phone is a personal entity and always with the user, the apps developed should be such that invariably interact, and prove expedient to the user.

Following are the survey findings on the key characteristics and features that differentiate mobile apps from conventional desktop applications. In order to be better understood, these characteristics are classified into three categories i.e. Hardware, Software and Communication.

\section{A. Characteristics related to Hardware}

Less Power (CPU, memory, and battery): Mobile apps are relatively small in size by lines of code therefore require less disk space. Mobile phones are still weaker than personal computers as they usually have less memory and weaker CPUs which drain faster when used for longer durations. The survey respondents believe that efficient code and good architectures are more important for mobile phones than for desktops. In addition, the respondents propose that mobile apps should be designed in such a manner that they consume less computing power and should run fast as well.

Input Mechanism: As per $60 \%$ of the respondents, input mechanism is a key characteristic that distinguishes mobile apps from desktop applications. The human input mechanisms in mobile apps differ from desktop applications because of the features such as touch, pinch, and swipe. It is recommended that a mobile application should be user and touch friendly with an option for more selections along with minimal keyboard entries. It is proposed that traditional keyboard typing input is more cumbersome and should be minimized. As a result, developers should not require the users to input substantial text, instead should allow them to input through a list of selectable options.

Screen Size and Form Factor: About 55\% of participants consider size, shape, style of a mobile phone, as well as its layout and position as a premium entity because a mobile device uses touch screen and small keypad to receive input, maintaining its small size. Since mobile screen size varies from one device to another, it is suggested that developers need to select the number of features they want to introduce well in advance when designing a smart phone application.

Start-up Time: Since mobile device users use mobile devices frequently and for short durations, the ability to quickly start up a mobile app is considered to be imperative by survey participants. The combination of the use contexts of being on the move and necessitating information quickly means that mobile apps need to be laid out differently to desktop applications.

Physical Parameters: Physical parameters, such as illumination, noise, temperature and humidity, vibration and motion, etc. of mobile users environments are extremely variable, limiting or excluding one or more modalities unlike desktop computers. Context has much deeper effects on interactions as people in mobile conditions devote very limited attention while interacting with applications on the device. Therefore, the survey respondents suggest that mobile apps should be designed for fast usage through few choices or decisions, for which a high degree of intelligence is expected from an mobile application.

Device Fragmentation: Unlike traditional software, around $33 \%$ of the respondents believe that mobile apps should run on all mobile platforms and be compatible with different device versions and their OS versions.

\section{B. Characteristics related to Software}

\section{Application Interaction}

User Experience (UX): Over $75 \%$ of the survey respondents confirm "user experience" as the deciding factor in mobile apps and prototype designing to be a crucial activity. In order to get an excellent user experience, a user should feel comfortable in gadget interaction and feel smart enough to accomplish any task with intuitive use, without any tutorial or additional help. For highly productive user experience and good mobile app designing, it is recommended to consider characteristics such as Quick Start-up time, 
responsiveness, and focused application purpose when designing, building, and testing a mobile app.

User Interface (UI): A mobile app should be designed to match the look-and-feel of the targeted mobile environment. User experience expertise and knowledge of the target mobile environments standards (both UI and code level) are important to provide users with a pleasant and consistent application. It is suggested by the survey respondents that services developed for mobile devices should take into account user interaction, situation and information required while the device is used. In addition, the respondents believe that functions such as "Back" and "Exit" option should be mandatory and must be included in a mobile app. Further, there should be closely guided usage paths that suggest the next step to a user, rather than offering multiple paths.

Interaction with Information Sources: Since a native mobile app requires interaction with information sources for data transfer, it must communicate with predefined content and data sources to fetch information and return results. According to $60 \%$ of the respondents, to build a good mobile app, a flexible and extensible set of connectors to quickly integrate a native mobile app and backend information sources should be provided by identifying the data sources, taking into consideration how frequently information is updated on the devices, and the extent of data that flows across the network.

Integration with other Apps: About 35\% of the respondents emphasize that integration with other apps and data on a user's device should be tested (wherever appropriate), and verified before the deployment and market release.

Action Feedback: The majority of the respondents are of the opinion that mobile users should get an 'acknowledgement' instantly upon performing an action. They believe that the worst response is that the device remains unresponsive and does nothing, and leaves users to speculate whether their action is recorded or not.

Error Notification: Approximately $10 \%$ of the respondents believe that error handling in mobile applications has to be effective such that it will have minimum effect on users and may be quickly noticed and communicated to users. The mobile user should be able to monitor and track all the key events and errors in the app, and get push notifications for problem alerts. Push notification allows an app to notify user of new messages or events without the need to actually open the application.

\section{Application Development}

Application Focus: About $60 \%$ of the survey respondents suggest that mobile apps should be simple, focused with specific features and should not try to do everything as opposed to desktop applications that offer a variety of features. Each app should have its own specific goals/objectives with a minimum number of clicks, taps or other user gestures and respond in a rapid way.

Experienced Resource: It is imperative to follow the style guidelines and behaviour of each specific device when building a mobile app. For this purpose, $20 \%$ of the respondents emphasize the necessity of experienced resources for the development of mobile apps, since they often offer only one way to accomplish a given task as compared to desktop applications that offer multiple ways for the successful completion of a given task.

Convenience: Mobile application development projects usually have deadlines and a quick turnaround time for market release. However, all requirements are not spelled out in the beginning and they vary considerably during the process. On the contrary customers expect developers to be agile enough and be able to make immediate changes in case of any hardware, software development, technology or other issues. To achieve this, an application needs to be developed within specific time-frame through effective team collaboration. However, it should be imperative that the app design be simple, ensuring good content, high value and acceptance among users.

Responsiveness: Many respondents advise developers to consider User Interface (UI) area, functionality, and databases access and make sure that an application remains responsive when running long operations such as database access, network access, and I/O.

Personalization: Creating individual content and role based on personalized context or usage is another characteristic especially when BYOD (Bring your own device) is considered. The survey respondents believe that users desire that the concerned app should fit their needs and perform the way they want. This specific attribute not only covers custom-made content but also controls over data that is stored, shared or used for more actions.

Localization: Unlike traditional software, some mobile apps handle sensors that respond to device movement, numerous gestures, global positioning system, cameras and multiple networking protocols. About $30 \%$ of the respondents believe that localized information and the possibility to provide position-based information are crucial features that render mobility impressive, convenient, valuable, and provide good user experience.

Reachability: Unlike traditional software, approachability in excellent mobile apps means that they can be used anywhere at any time. It is true for mobile apps that reachability has become full time availability in terms of updated information and continuous usefulness.

\section{Application Security}

Around $20 \%$ of the survey respondents point out that unlike traditional software, errors usually occur in session management when building a secure mobile app. They believe that these errors should be rectified since they may prove vulnerable to mobile apps.

Encryption: As security plays a vital role when designing a mobile app, data transferred over a network must be encrypted through the carrier network as some apps sync data with online. To prevent attackers from easily gaining access to valuable information and resources on a mobile device, Secure Sockets Layer (SSL) may be utilized to encrypt the information of a client and require a mobile certificate that can be validated. 
Expire Sessions: Like traditional applications, mobile developers normally allow a mobile app session to remain active for a longer period. Attackers may exploit this fact to make malicious requests to the server as long as the session is active. According to the respondents, mobile developers should consider this as a threat since they are trading away security in favour of a minor convenience to users.

Request Validity Period: Unlike traditional applications, there is a greater possibility of attacker intercepting and modifying information as long as a request is valid. The survey respondents suggest mobile developers to limit and allow automatic application session outs. All requests should be time stamped on the client side and should expire on the server side after a defined period of time.

Prevent Repeat Request: There is a particular privacy and customizability need for mobile users. In order to build a robust mobile app, as per the respondents, developers must check the measures to prevent repeated requests as attackers can replay intercepted requests. Access to certain sensors like geo-location and private data typically requires approval but once approved, users have no additional control and believe the application is performing optimally. If a mobile app has free access to the network, it can call any host and exchange any information without user notification. In terms of access to information, this constraint and ability differs mobile platforms from desktops.

\section{Characteristics related to Communication}

Network Connectivity: As per 55\% of the survey respondents, common mobile network connection properties are low bandwidth, high latency, unstable connections, data transfer charges and battery intensive. Mobile devices use a variety of ways to connect to the Internet, ranging from GPRS to $\mathrm{Wi}-\mathrm{Fi}$. Since some mobile apps are always online, there may be unstable, intermittent or unreliable connectivity as a user moves in and out of cell coverage. And even if a mobile device is always connected, the speed and quality of a network vary riotously.

\section{DISCUSSION AND CONCLUSION}

In general, desktop applications are used in long sessions that offer an exploratory environment. In contrast, good mobile apps offer a focused and efficient experience for accomplishing specific tasks in short session durations. It is important that the mobile development community understands these concepts along with the specific technologies.

Very few scientific publications can be found which specifically identify key characteristics and what makes mobile applications different from traditional software. Considerable work still remains to be carried out in this area in terms of further validation of the underlying hypotheses, and in-depth explanation of each characteristic.
The findings of this study are summarized in Table 3 by classifying the key characteristics that differentiate mobile apps from traditional applications into three categories: characteristics related to Hardware, Software (application interaction, application development, and application security) and Communication.

Table 3. Characteristics of Mobile Applications

\begin{tabular}{|c|}
\hline Characteristics related to Hardware \\
\hline Less Power \\
\hline Input Mechanism \\
\hline Screen Size and Form Factor \\
\hline Start-up Time \\
\hline Physical Parameters \\
\hline Device Fragmentation \\
\hline Characteristics related to Software \\
\hline Application Interaction \\
\hline User Experience \\
\hline User Interface \\
\hline Interaction with Information Sources \\
\hline Integration with other Apps \\
\hline Action Feedback \\
\hline Error Notification \\
\hline Application Development \\
\hline Application Focus \\
\hline Experienced Resource \\
\hline Convenience \\
\hline Responsiveness \\
\hline Personalization \\
\hline Localization \\
\hline Reachability \\
\hline Application Security \\
\hline Encryption \\
\hline Expire Sessions \\
\hline Request Validity Period \\
\hline Prevent Repeat Request \\
\hline Characteristics related to Communication \\
\hline Network Connectivity \\
\hline
\end{tabular}

The limitations of the study lie in the fact that the responses were received only from people willing to be surveyed. There is a risk of getting responses from people with strong feelings about the area and the survey questions captured opinions, not facts. The biases of the communities may be reflected in the results. Due to the same fact, the survey sample may not represent the real population of the mobile application development community, Therefore the generalizability of the findings need to be further tested by future work.

In conclusion, we strongly believe that this paper will provide relevant contribution. Practitioners can benefit from our analysis in order to better understand the current trends in the mobile application development. We are conscious that this paper does not address all the identified characteristics and functions in depth. We hope 
that the findings will offer some guidance for further academic research. In addition, to put the findings of this study into perspective, a systematic comparison to software development in the domains other than mobile industry could be conducted in future research.

\section{REFERENCES}

[1] White Paper "Software Testing Garbage", [Online: http://softwaretestinggarbage.blogspot.in/2012/10/typesof-mobile-application.html], 2012.

[2] Viktor Kolokolov, Paul Baumann, Silvia Santini, Stefan T. Ruehl, Stephan A. W. Verclas, "Flexible development of variable software features for mobile business applications", Workshops Proceedings of the 17th International Software Product Line Conference (SPLC'13), pp. 67-73, 2013.

[3] Anthony Wasserman, "Software engineering issues for mobile application development", FSE/SDP workshop on Future of software engineering research (FoSER'10), ACM, 397-400, 2010.

[4] I. Salmre, "Characteristics of Mobile Applications", [Online:http://ptgmedia.pearsoncmg.com/images/0321269 314/samplechapter/salmre_ch02.pdf], pp. 19-36, 2004.

[5] Qiang Pu, Ahmed Lbath, Daqing He, "Location Based Recommendation for Mobile Users Using Language Model and Skyline Query", International Journal of Information Technology \& Computer Science (IJITCS), vol. 4, no.10, pp. 19-28, 2012.

[6] Josh Dehlinger, Jeremy Dixon, "Mobile Application Software Engineering: Challenges and Research Directions", Proceedings of the Workshop on Mobile Software Engineering, Springer, pp. 29-32, 2011.

[7] Gordon AJ, "Concepts for mobile programming", Proceedings of the 18th ACM conference on Innovation and technology in computer science education (ITiCSE), ACM, pp. 58-63, 2013.

[8] Marius POPA, "Audit Process during Projects for Development of New Mobile IT Applications", Informatica Economica, vol. 14 no. 3, pp. 34-46, 2010.

[9] Andreou, A.S., Panayidou, D., Andreou, P., Pitsillides, A. "Preserving Quality in the Development of Mobile Commerce Services and Applications", ACIT Software Engineering, Novosibirsk, Russia, pp. 11-16, June 2005.

[10] Paul Pocatilu, Catalin Boja, "Quality Characteristics and Metrics Related to M-learning Process", Amfiteatru Economic, vol. 11, pp. 346-354, 2009.

[11] Garofalakis, J., Stefani, A., Stefanis, V., \& Xenos, M., "Quality attributes of consumer-based m-commerce systems", IEEE International Conference on E-Business and Telecommunication Networks, pp. 130-136, 2007.

[12] Shamsudeen Rabi, Akanmu Semiu, Okere P. Hector, "Usability characteristics of mobile applications," Proceedings of International Conference on Behavioural \& Social Science Research (ICBSSR), Kampar, Malaysia, pp. 1-5, Nov 2012.

[13] Mobile, W., Are, S., \& Sites, D. W. "10 Ways Mobile Sites Are Different from Desktop Web Sites", [Online: www.uxmatters.com/mt/archives/2011/03/10-waysmobile-sites-are-different-from-desktop-web-sites.php], 2011.

[14] James B. Zimmerman, "Mobile Computing: Characteristics, Business Benefits, and the Mobile Framework", Technical Report INSS 690 CC, University of Maryland European Division-Bowie State, pp. 1-27, Apr 1999.
[15] Deepak G. and B. S. Pradeep, "Challenging Issues and Limitations of Mobile Computing", International Journal of Computer Technology \& Applications (IJCTA), vol. 3, no. 1, pp. 177-181, 2012.

[16] Adrian Holzer \& Jan Ondrus "Mobile application market: A developer's perspective", Telematics and Informatics, vol. 28 no. 1, pp. 22-31, Feb 2011.

[17] Harleen K. Flora, Swati V. Chande, Xiaofeng Wang, "An Investigation into Mobile Application Development Processes: Challenges and Best Practices" International Journal of Modern Education and Computer Science (IJMECS), vol. 6, pp. 1-9, 2014.

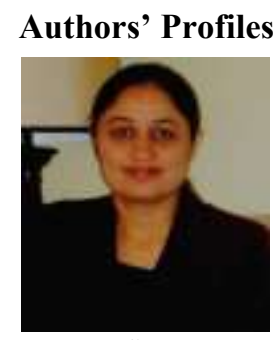

Harleen K. Flora is a certified PMP, ITIL Project Manager and holds Masters degree in Computer Management from University of Pune, India. She has over 15 years of extensive experience in designing, developing and managing software development projects. She worked for Lockheed Martin and served many US government projects as a Consultant. Her research areas include Agile software development methodologies and practices, mobile application development process and software engineering.

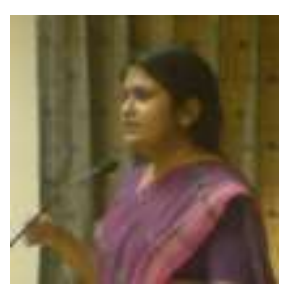

Dr. Swati Chande has over 22 years of experience in teaching, industry, and research. She also has extensive corporate and technical training experience. She completed her M.S. in Software Systems from Birla Institute of Technology and Science, Pilani, Doctorate from Banasthali University, and also has a M.Sc. in Mathematics. Her research interests include database management, genetic algorithms, computational thinking, and software engineering.

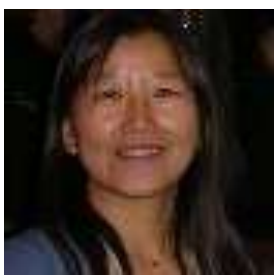

Dr. Xiaofeng Wang is a researcher at the Free University of Bozen-Bolzano, Italy. Her research areas include software development process, methods, agile software development, and complex adaptive systems theory.

How to cite this paper: Harleen K. Flora, Xiaofeng Wang, Swati V. Chande,"An Investigation on the Characteristics of Mobile Applications: A Survey Study", International Journal of Information Technology and Computer Science(IJITCS), vol.6, no.11, pp.21-27, 2014. DOI: 10.5815/ijitcs.2014.11.03 